\title{
On the Influence of Autonomy and Transparency on Blame and Credit in Flawed Human-Robot Collaboration
}

\author{
Alexander Arntz \\ alexander.arntz@hs-ruhrwest.de \\ University of Applied Sciences Ruhr West \\ Bottrop, North-Rhine Westphalia, Germany \\ Carolin Straßmann \\ University of Applied Sciences Ruhr West \\ Bottrop, Germany \\ carolin.strassmann@hs-ruhrwest.de
}

\author{
Sabrina C. Eimler \\ University of Applied Sciences Ruhr West \\ Bottrop, Germany \\ sabrina.eimler@hs-ruhrwest.de \\ H. Ulrich Hoppe \\ University of Duisburg-Essen \\ Duisburg, Germany \\ hoppe@collide.info
}

\begin{abstract}
The collaboration between humans and autonomous AI-driven robots in industrial contexts is a promising vision that will have an impact on the sociotechnical system. Taking research from the field of human teamwork as guiding principles as well as results from human robot collaboration studies this study addresses open questions regarding the design and impact of communicative transparency and behavioral autonomy in a human robot collaboration. In an experimental approach, we tested whether an AI-narrative and communication panels of a robot-arm trigger the attribution of more human like traits and expectations going along with a changed attribution of blame and failure in a flawed collaboration.
\end{abstract}

\section{CCS CONCEPTS}

- Human-centered computing $\rightarrow$ Empirical studies in collaborative and social computing.

\section{KEYWORDS}

human-robot collaboration; attribution of blame; perception of intelligence; online study

\section{ACM Reference Format:}

Alexander Arntz, Sabrina C. Eimler, Carolin Straßmann, and H. Ulrich Hoppe. 2021. On the Influence of Autonomy and Transparency on Blame and Credit in Flawed Human-Robot Collaboration. In Companion of the 2021 ACM/IEEE International Conference on Human-Robot Interaction (HRI '21 Companion), March 8-11, 2021, Boulder, CO, USA. ACM, New York, NY, USA, 5 pages. https://doi.org/10.1145/3434074.3447196

\section{INTRODUCTION}

Industrial usage of Human-Robot Collaboration (HRC) is expected to be enhanced by advanced artificial intelligence (AI) technology in the future, enabling robots to operate either partially or fully autonomously in conjunction with the employees [22]. Whereas current implementations of HRC in industrial settings often use

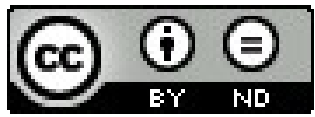

This work is licensed under a Creative Commons Attribution-NoDerivs International 4.0 License.

HRI '21 Companion, March 8-11, 2021, Boulder, CO, USA. (C) 2021 Copyright held by the owner/author(s).

ACM ISBN 978-1-4503-8290-8/21/03. https://doi.org/10.1145/3434074.3447196 non-humanoid robots (e.g. robot-arms) controlled by an operator or following predefined routines, an autonomous AI-driven robot, capable of adapting and reacting in the required task, would embrace the full potential of the HRC concept [2]: Human personnel could be relieved by delegating repetitive or heavy work onto the robot, while contributing through intuition and experienced-based decision-making, thus combining the advantages of both parties [11]. However, besides the technical and safety challenges to be solved, there are reservations against $\mathrm{AI}$ and robots in large parts of the general population [29] that need careful investigation. Prior research in the realm of e.g. the Media Equation Theory [27] and CASA [26] has shown that individuals tend to project human characteristics onto robots while interacting with them and that this even holds true for technologies with non-humanoid appearance $[9,28]$. Therefore, it can be assumed that principles and results obtained in research on group collaboration might be applicable for HRC as well.

Comparable to collaboration among human personnel, the collaboration with an autonomous robot to achieve a common goal creates an interdependency in the work relation [8]. Errors in such a context can hamper the successful outcome of the procedure. Considering they might lead to costly or hazardous ramifications for the human collaboration partner, it is of interest to investigate if an autonomous robot will be held accountable for an error and which characteristics along with behavior can influence people's attribution of blame. Research that examined the accusation of errors in non-work-related, casual scenarios [24], revealed that more autonomy displayed by a robot-arm results in an increased attribution of errors whereas comprehensibility/transparency of the robot's actions leads to a decrease in blame [17]. However, the attribution of accountability and credit made in HRC-workplace environments involving autonomous robots remains an open question. Also, reservations towards AI need further exploration to design successful and accepted HRC scenarios, especially attributions relevant in collaborative tasks (e.g. manufacturing procedures) are worth being researched.

To explore this, we used the virtual reality sandbox application by [5] that is capable of simulating a variety of industrial HRC scenarios difficult to realize under experimental conditions [20]. The environment contained a robot-arm with autonomous behavior serving as a basis for an online study testing using non-interactive videos, in a collaborative task, the influence of a) the robot-arms 
behavioral autonomy and b) its transparency in communication on the attribution of blame and credit.

\section{RELATED WORK}

Research addressing the attribution of blame and credit is well established in the field of human group collaboration. The self-serving bias in attribution has been identified as the main contributing factor in people's assessment of outcomes [16]. Two types can be distinguished: the internal attribution, that includes the own characteristics of an individual, and the external attribution, containing outside influences [12]. Internal attribution is often associated with successful outcomes whereas people incline to apply external attribution to poor outcome [23]. Studies involving Human-Robot Interaction showed that this behavior also occurs when people engage with robots $[10,13,17]$. This misattribution can negatively affect trust in the robot's capability to accomplish a task, thus mitigating the collaboration process [15]. While the self-serving bias in attribution provides a strong foundation, contrary to prior studies, Lei and colleagues' participants attributed more credit and less blame to the robot [19]. The studies used divergent representations of robots with different levels of autonomy and communication, which might have contributed to the inconsistent results. Accordingly, the design of the robot has an effect on the described attribution process (compare [17]). To design robots to be the best possible collaboration partners and bypass distrust [15], research is needed to understand which characteristics drive the attribution of blame in the collaboration with robots.

\subsection{The Effect of Expected Autonomy}

Kim and colleagues [17] demonstrated an influence of the robot's autonomy on the attribution of blame. A robot that presented more autonomy was more likely blamed for an undesirable outcome. This is specifically of interest in industrial collaboration settings, where employees often have misconceptions and negative attitudes towards autonomous systems [29]. Especially the term AI is associated with negative feelings [1], since people are afraid that robots with AI-capabilities will take their jobs. As a consequence of this widespread misconceptions about $\mathrm{AI}$ among the general population, people tend to attribute various forms of human-like characteristics and behavior towards such systems [21]. This is also plausible against the background of CASA [26]. It is therefore assumed that the introduction of the term AI along with the autonomous behavior by a robot-arm will invite participants to project more human-like abilities and behavior onto the system [30]. As a result, people will use an external attribution and blame the robot with higher expected autonomy more for errors and negative outcomes of a collaboration process. Accordingly, the following hypotheses are assumed:

H1: Participants attribute more human-like abilities (intelligence, morality) to a robot-arm with AI-capabilities compared to one without.

H2: Participants attribute more blame and less credit to a robotarm with AI-capabilities compared to one without.

\subsection{The Effect of Communication and Transparency}

Beside the perceived autonomy and intelligence of the robot, transparency was found to affect the attribution of blame [17]. A robot that explains it's own behavior was found to evoke lower attributions of blame. This might also an explanation for the results of Lei and colleagues, since they tested the attribution of the talking humanoid robot NAO [19]. Communicative behavior that elicits transparency of the robot's behavior, seems to prevent external attribution. In industrial settings robot-arms are often limited in their communicative abilities. As the environment is often loud, verbal outputs would not work. Studies therefore suggest to use text-panels to enrich the communicative output of the robot. Making the robot's behavior transparent to the human collaborator by augmenting the robot with communication capabilities was found to result in various benefits, e.g. perceived stress and general, positive emotions on the side of the human collaborator $[4,5]$. Accordingly, we assume that an communication panel affects the perception of the robot as collaboration partner and thereupon leads to fewer external attributions of errors. This leads to the following hypotheses:

H3: Participants perceive a robot-arm equipped with a communication panel as better collaboration partner (more cooperative and better quality of the collaboration) than one one without communication ability.

H4: Participants attribute less blame and more credit to a robotarm equipped with a communication panel compared to one without.

As described above, studies involving communicative robots often use voice output that distinctly link the statements to the respective robot [17, 19]. However, industrial robots with non-humanoid appearances in extremely loud environments demand different communication channels. A prior study recognized text-panels in natural language as a viable means of communication in industrial HRC settings [3]. Results indicated that proximity and visual relation to the robot-arm are decisive aspects, since the external text statements are not as intuitively assignable to the robot. Only when the communication behavior (text-panel) is assigned to the robot, it is plausible that this affects the attribution process of errors and robot perception. It is therefore of interest if participants associate the text-panels to the robot-arm or whether it is perceived as an other autonomous entity. Thus, the following research question is to be answered:

RQ1: Do participants see the text-panel augmentations as part of the robot-arm or as another autonomous entity?

\section{METHOD}

For the study, we used a virtual reality simulation of a HRC sharedtask setup as described by [3]. Since the pandemic made a VR-lab experiment impossible, an online experiment was set up, in which participants were presented a first-person perspective video of the HRC-setup. In a 2 (augmented communication vs. non-augmented condition) x 2 (AI-narrative vs. non-AI-narrative) between-subjects design participants were asked to imagine themselves in the role of the human worker assigned to the HRC working arrangement. A total of 225 participants took part in the online study. Participants 
under 18, an outlier aged 99 as well as participants completing the experiment too fast or too slow (i.e. deviating 1.5 standard deviations from mean completion time) were excluded. Altogether 34 were sorted out. The average age of the remaining 191 participants was $M=25.12(S D=7.51)$. 91 were female, 97 male and 3 non-binary people participated.

\subsection{Material}

Participants were exposed to one of the four conditions. In all conditions participants a virtual representation of a LBR iiwa 7 R800 CR robot-arm was displayed that used multi colored light-signals and action initiating/terminating and standby gestures $[5,18,25]$. In the high-transparency condition (= augmented condition) text-panels in natural language were used to express guidance and explanations. In the low transparency condition (= non-augmented condition), the explanatory and guiding text-panels were omitted, while everything else to be witnessed in the procedure was identical in terms of movement and actions by the robot and the human. The purpose for removing the text-panel, was to withhold the explanation of the robot-arm's behavior provided by the text-panel but retain the other communication methods. This maintained the robot-arm's ability to convey a detected error but obscure the system's interpretation of the error. To manipulate different levels of autonomy, participants were either told that the robot-arm has AI-capabilities or the scene was just depicted as a collaboration between a human worker and a robot-arm. In all conditions, participants witnessed, from a first-person perspective, a simulated shared-task in which they, as the human operator, were tasked to manufacture metal buttons through a press with their robot collaboration partner [5] During the procedure, both partners deviated from an assembling procedure and made two recognizable errors by either performing the wrong working step or violating the safety distance, causing a delay in the execution of the procedure.

\subsection{Measures and Procedure}

The online study was set up on the soscisurvey platform. After providing informed consent, participants were exposed to one of the four conditions. A text of their respective condition either told them that the robot in the collaboration scenario was equipped with AI-capabilities or just referred to as a collaboration with a robot-arm. The subsequent video either showed the augmented or non-augmented collaboration scenario. After being exposed to the stimulus, participants rated their attribution of blame to the robot (2 items, $\alpha=.820$ ) and to the self for the errors during the assembling task ( 2 items, $\alpha=.820$ ), as well as regarding the attribution of credit to the robot (2 items, $\alpha=.616$ ) and the self for task completion (2 items, $\alpha=$.625) [17]. The Perceived Moral Agency scale by [6] was used to assess morality (6 items, $\alpha=.763$ ) and dependency (4 items, $\alpha=.683$ ). Embodiment of the robot-arm was assessed through the EmCorp-Scale [14], containing the sub-scales: corporeality (3 items, $\alpha=$.685), expressiveness ( 4 items, $\alpha=.701$ ), tactile interaction $\&$ mobility (6 items, $\alpha=.539)$ and perception \& interpretation $(7$ items, $\alpha=.765$ ). To analyze the anthropomorphism (5 items, $\alpha=$ .592 ), animacy (5 items, $\alpha=.661$ ), likeability (5 items, $\alpha=.815$ ) and perceived intelligence ( 5 items, $\alpha=.761$ ) of the robot-arm, the questionnaire incorporated the Godspeed-scale by [7]. Moreover, the collaboration success was measured with an ad-scale consisting of 6 items $(\alpha=.795)$. The assessment of the components assigned to the robot was realized through screenshots of the application, where every visible item was highlighted through a bounding box. For each component participants were asked to decide whether or not it belonged to the robot. The questionnaire closed with demographics (e.g. age, gender, job position, educational background).

\section{RESULTS}

To test the hypotheses, multiple analyses of variance (MANOVA and ANOVA) were run including the relevant independent and dependent variables for testing.

H1: Participants attribute more human-like abilities (intelligence, morality) to a robot-arm with AI-capabilities compared to one without.

The analysis did not indicate significant differences in the attribution of intelligence and perceived morality between both AIconditions. Thus, the AI-narrative did not lead to higher perceived intelligence or higher attribution of moral capabilities. The robotarm was rated significantly better in the ability to perceive and interpret its surroundings $\left(F(1,187)=5.70, p=0.018, \eta_{p}^{2}=0.03\right)$ in the AI-narrative condition $(M=2.37, S D=.66)$ compared to the non-AI-narrative $(M=2.14, \mathrm{SD}=.73)$. Moreover, the capacity for cooperation is rated significantly better $(F(1,187)=6.47, p=$ $\left.0.012, \eta_{p}^{2}=0.03\right)$ in the AI-narrative condition $(M=3.91, S D=.92)$ compared the non-AI-narrative $(M=3.55, S D=1.11)$. Furthermore, the robot-arm was rated as significantly less dependent on predefined programming $\left(F(1,187)=5.92, p=0.016, \eta_{p}^{2}=0.03\right)$ in the AI-narrative condition $(M=4.22, S D=.74)$ compared to the nonAI-narrative $(M=4.46, \mathrm{SD}=.60)$. Although the AI-narrative did not lead to more perceived intelligence and morality, these results indicate that participants associate more human-like characteristics to the robot-arm like being independent, cooperative and able to perceive and interpret. Thus, H1 is partly supported.

H2 \& H4: Participants attribute more blame and less credit to a robot-arm with AI-capabilities compared to one without and participants attribute less blame and more credit to a robot-arm equipped with a communication panel compared to one without.

Analyses testing this did not show any differences between the conditions for neither the attribution of blame nor for credit attribution. Therefore, $\mathrm{H} 2$ and $\mathrm{H} 4$ could not be supported.

H3: Participants perceive a robot-arm equipped with a communication panel as better collaboration partner (more cooperative and better quality of the collaboration) than one one without communication ability.

Results show significant differences $(F(1,187)=5.95, p=0.016$, $\left.\eta_{p}^{2}=0.003\right)$ between the augmented condition $(M=3.57, S D=$ $.62)$ and the non-augmented condition $(M=3.33, S D=.76)$ for perceived intelligence. Significant differences occurred for dominance $\left(F(1,187)=8.67, p=0.004, \eta_{p}^{2}=0.04\right)$. The robot-arm in the augmented condition was perceived as more dominant $(M=3.37, S D=$ $.94)$ than in the non-augmented condition $(M=2.98, S D=.84)$. A significant difference was found for collaboration success $(F(1,186)$ $\left.=5.34, p=0.002, \eta_{p}^{2}=0.03\right)$, augmented condition $(M=2.80, S D=$ $.72)$ vs. non-augmented $(M=3.05, S D=.77)$. H3 is supported. 


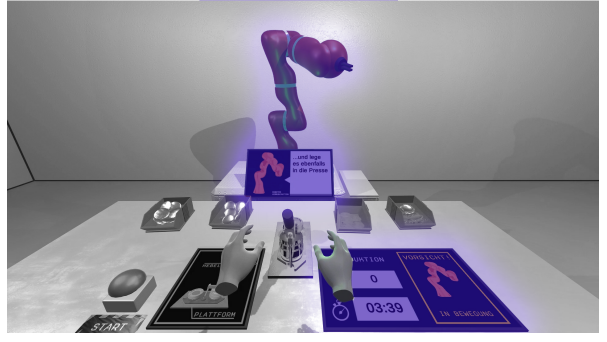

(a) Text-panel condition

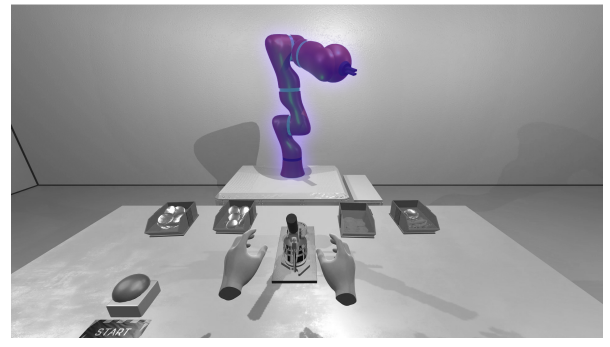

(b) Non-text-panel condition

Figure 1: Heat map of the components that participants associated with the robot-arm. The text-panels from the robotarm were considered part of the robot-arm.

RQ1: Do participants see the text-panel augmentations as part of the robot-arm or as another autonomous entity?

Analyzing the components that were associated with the robot, no significant differences were observed between the AI-narrative condition vs. non-AI-narrative condition. A heat map revealed that participants from all conditions identified the body of the robot-arm (Fig 1). In addition, 93.3\% of the participants from the text-panel condition associated the text-panel as part of the collaboration partner. Also, $73.2 \%$ of the participants associated the information text-panel featuring warning messages with the robot-arm.

\section{DISCUSSION AND LIMITATIONS}

This study explored the effect of transparency (i.e. communicative augmentations) and autonomy (i.e. AI-narrative) on the attribution of blame and credit as well as the general perception of the robot and the collaboration in an industrial HRC assembly task setting. Although the introduction of the AI-term and narrative did not lead to a higher attribution of intelligence and morality to the robot it invoked associations with other human-like characteristics (H1): Participants rated the robot-arm as more capable of perceiving and interpreting its surroundings and noted a greater ability for cooperation when they believed it to be equipped with AI. Considering that participants underwent the same procedure and witnessed the same behavior of the robot-arm in all conditions, backed by the research of [30], it can be assumed that participants projected their own mental models and expectations of the AI-term onto the characteristics of the robot-arm. As stated by [30], the wide spectrum of the term AI together with the widespread misconceptions invites people with media biased knowledge to project numerous abilities and expectations onto AI-enhanced systems [29]. Future studies should explore the content of the mental models and expectations and their effects on the collaboration process.

RQ1 investigated which components are attributed to the robotarm to ensure the statements displayed on the text-panel is associated with the robot-arm. Indeed participants perceived the text-panel as belonging to the robot/ part of the robot-arm. Accordingly, the presence of text-panels as a means for the robot to increase communicative transparency lead to higher attributions of intelligence, dominance and the perception of a more successful collaboration (H3). Although participants perceived the collaboration more successful in the augmented version of the robot-arm, the text-panel did not affect the evaluation of the robot-arm's perceived cooperativeness. In contrast, the augmented robot-arm was perceived as more dominant. This results could produce conflicts, since the perception of dominance elicits negative feelings in the human collaborator. Especially people with fears and negative attitudes might feel patronized and avoid collaborating with the robot-arm. Thus, designers have to use communication features inducing transparency with caution, since they could trigger a boomerang-effect.

While the perception of the robot-arm was affected by the induced autonomy and transparency (H1, H3), no significant difference regarding blame and credit was found (H2, H4). While other studies showed that the self-serving bias in attribution cannot always be demonstrated in interactions with robots [19], the projected ramification that is expected by the individual [16] must be considered as a limitation of this study.

Due to the restrictions of the COVID-19 pandemic we were unable to conduct an experimental study where participants could actually collaborate with robot-arm in the virtual reality scenario. While the virtual reality sandbox application provided by [5] enabled us to substitute an online study design, no direct interaction with the robot-arm was possible. The immersive effect of the environment might be able to create a sense of more direct involvement with a higher sensitivity for the outcomes of the errors happening. Employees exposed to autonomous robots in industrial HRC settings could face real consequences from errors made during the collaboration e.g. injury or career disadvantages that witnessing a video cannot fully mimic. Future work should address the used scenario using an interaction study to overcome these limitations. Also, future studies should investigate if additional communication augmentations (e.g. voice output) and inputs provided by the text panels affect attributions differently.

\section{CONCLUSION}

While this study could not replicate established findings from the literature regarding the attribution of blame and credit, results reveal an interesting effect regarding the attribution of human characteristics on the robot-arm caused by an AI-narrative that are worth being further explored. Future studies should look into the dynamics of people's mental models and preconceptions brought into the collaboration scenario with AI-based robots. Communication behavior should be outbalanced in a way that it does not trigger dominance perception on the one hand, but elicits enough transparency to induce trust in the collaboration partner on the other hand. 


\section{REFERENCES}

[1] 2017. Attitudes towards the impact of digitisation and automation on daily life: Report. Special eurobarometer, Vol. 460. [European Commission], [Brussels].

[2] Arash Ajoudani, Andrea Maria Zanchettin, Serena Ivaldi, Alin Albu-Schäffer, Kazuhiro Kosuge, and Oussama Khatib. 2018. Progress and prospects of the human-robot collaboration. Autonomous Robots 42, 5 (2018), 957-975. https: //doi.org/10.1007/s10514-017-9677-2

[3] Alexander Arntz and Sabrina C. Eimler. 2020. Experiencing AI in VR: A Qualitative Study on Designing a Human-Machine Collaboration Scenario. In $\mathrm{HCI}$ International 2020 - Late Breaking Posters, Constantine Stephanidis, Margherita Antona, and Stavroula Ntoa (Eds.). Communications in Computer and Information Science, Vol. 1293. Springer International Publishing, Cham, 299-307. https://doi.org/10.1007/978-3-030-60700-5 38

[4] Alexander Arntz, Sabrina C. Eimler, and H. Ulrich Hoppe. 14.12.2020 - 18.12.2020. "The Robot-Arm Talks Back to Me" - Human Perception of Augmented HumanRobot Collaboration in Virtual Reality. In 2020 IEEE International Conference on Artificial Intelligence and Virtual Reality (AIVR). IEEE, 307-312. https://doi.org/ 10.1109/AIVR50618.2020.00062

[5] Alexander Arntz, Sabrina C. Eimler, and H. Ulrich Hoppe. 2020. Augmenting the Human-Robot Communication Channel in Shared Task Environments. In Collaboration Technologies and Social Computing, Alexander Nolte, Claudio Alvarez, Reiko Hishiyama, Irene-Angelica Chounta, María Jesús Rodríguez-Triana, and Tomoo Inoue (Eds.). Lecture Notes in Computer Science, Vol. 12324. Springer International Publishing, Cham, 20-34. https://doi.org/10.1007/978-3-030-58157-2\{_\}2

[6] Jaime Banks. 2019. A perceived moral agency scale: Development and validation of a metric for humans and social machines. Computers in Human Behavior 90 (2019), 363-371. https://doi.org/10.1016/j.chb.2018.08.028

[7] Christoph Bartneck, Dana Kulić, Elizabeth Croft, and Susana Zoghbi. 2009. Measurement Instruments for the Anthropomorphism, Animacy, Likeability, Perceived Intelligence, and Perceived Safety of Robots. International fournal of Social Robotics 1, 1 (2009), 71-81. https://doi.org/10.1007/s12369-008-0001-3

[8] César A. Collazos, Luis A. Guerrero, José A. Pino, and Sergio F. Ochoa. 2003. Collaborative Scenarios to Promote Positive Interdependence among Group Members. In Groupware: Design, Implementation, and Use, Gerhard Goos, Juris Hartmanis, Jan van Leeuwen, Jesús Favela, and Dominique Decouchant (Eds.) Lecture Notes in Computer Science, Vol. 2806. Springer Berlin Heidelberg, Berlin, Heidelberg, 356-370. https://doi.org/10.1007/978-3-540-39850-9\{_\}30

[9] Stéphanie Dubal, Aurélie Foucher, Roland Jouvent, and Jacqueline Nadel. 2011 Human brain spots emotion in non humanoid robots. Social cognitive and affective neuroscience 6, 1 (2011), 90-97. https://doi.org/10.1093/scan/nsq019

[10] Victoria Groom, Jimmy Chen, Theresa Johnson, F. Arda Kara, and Clifford Nass. 02.03.2010 - 05.03.2010. Critic, compatriot, or chump?: Responses to robot blame attribution. In 2010 5th ACM/IEEE International Conference on Human-Robot Interaction (HRI). IEEE, 211-217. https://doi.org/10.1109/HRI.2010.5453192

[11] Buxbaum Hans-Jürgen. 2020. Human-Robot Collaboration. International fournal of Robotic Engineering 5, 1 (2020). https://doi.org/10.35840/2631-5106/4121

[12] Fritz. Heider. 2013. The Psychology of Interpersonal Relations. Psychology Press. https://doi.org/10.4324/9780203781159

[13] Pamela J. Hinds, Teresa L. Roberts, and Hank Jones. 2004. Whose Job Is It Anyway? A Study of Human-Robot Interaction in a Collaborative Task. Human-Computer Interaction 19, 1-2 (2004), 151-181. https://doi.org/10.1080/07370024.2004.9667343

[14] Laura Hoffmann, Nikolai Bock, and Astrid M. v.d. Rosenthal Pütten. 02262018 The Peculiarities of Robot Embodiment (EmCorp-Scale). In Proceedings of the 2018 ACM/IEEE International Conference on Human-Robot Interaction, Takayuki Kanda, Selma Sabanović, Guy Hoffman, and Adriana Tapus (Eds.). ACM, New York, NY, USA, 370-378. https://doi.org/10.1145/3171221.3171242

[15] Poornima Kaniarasu and Aaron M. Steinfeld. 25.08.2014 - 29.08.2014. Effects of blame on trust in human robot interaction. In The 23rd IEEE International
Symposium on Robot and Human Interactive Communication. IEEE, 850-855. https://doi.org/10.1109/ROMAN.2014.6926359

[16] Harold. H. Kelley and John. L. Michela. 1980. Attribution theory and research. Annual review of psychology 31 (1980), 457-501. https://doi.org/10.1146/annurev. ps.31.020180.002325

[17] Taemie Kim and Pamela Hinds. 06.09.2006 - 08.09.2006. Who Should I Blame? Effects of Autonomy and Transparency on Attributions in Human-Robot Interaction. In ROMAN 2006 - The 15th IEEE International Symposium on Robot and Human Interactive Communication. IEEE, 80-85. https://doi.org/10.1109/ ROMAN.2006.314398

[18] Ingo Kresse. 2010. licensed under CC-BY-3.0 2010 (c) by Ingo Kresse, kresse@in.tum.de. https://creativecommons.org/licenses/by/3.0/.

[19] Xin Lei and Pei-Luen Patrick Rau. 2020. Should I Blame the Human or the Robot? Attribution Within a Human-Robot Group. International fournal of Social Robotics (2020). https://doi.org/10.1007/s12369-020-00645-w

[20] Séverin Lemaignan, Marc Hanheide, Michael Karg, Harmish Khambhaita, Lars Kunze, Florian Lier, Ingo Lütkebohle, and Grégoire Milliez. 2014. Simulation and HRI Recent Perspectives with the MORSE Simulator. In Simulation, Modeling, and Programming for Autonomous Robots, Davide Brugali, Jan F. Broenink, Torsten Kroeger, and Bruce A. MacDonald (Eds.). Lecture Notes in Computer Science, Vol. 8810. Springer International Publishing, Cham, 13-24. https://doi.org/10. 1007/978-3-319-11900-7\{_\}2

[21] Gabriel Lima, Changyeon Kim, Seungho Ryu, Chihyung Jeon, and Meeyoung Cha. 2020. Collecting the Public Perception of AI and Robot Rights. Proceedings of the ACM on Human-Computer Interaction 4, CSCW2 (2020), 1-24. https: //doi.org/10.1145/3415206

[22] Ruikun Luo, Rafi Hayne, and Dmitry Berenson. 2018. Unsupervised early prediction of human reaching for human-robot collaboration in shared workspaces. Autonomous Robots 42, 3 (2018), 631-648. https://doi.org/10.1007/s10514-017-9655-8

[23] Dale T. Miller and Michael Ross. 1975. Self-serving biases in the attribution of causality: Fact or fiction? Psychological Bulletin 82, 2 (1975), 213-225. https: //doi.org/10.1037/h0076486

[24] Tomohito Miyake, Yuji Kawai, Jihoon Park, Jiro Shimaya, Hideyuki Takahashi, and Minoru Asada. 14.10.2019 - 18.10.2019. Mind perception and causal attribution for failure in a game with a robot. In 2019 28th IEEE International Conference on Robot and Human Interactive Communication (RO-MAN). IEEE, 1-6. https: //doi.org/10.1109/RO-MAN46459.2019.8956325

[25] MORSE. 2020. KUKA LWR arm actuator. http://www.openrobots.org/morse/ doc/0.5/user/actuators/kuka_lwr.html\#use- of-the-kuka-lwr

[26] Clifford Nass, Jonathan Steuer, and Ellen R. Tauber. 1994. Computers are social actors. In Conference companion on Human factors in computing systems - CHI '94, Catherine Plaisant (Ed.). ACM Press, New York, New York, USA, 204. https: //doi.org/10.1145/259963.260288

[27] Byron Reeves and Clifford Ivar Nass. 1998. The media equation: How people treat computers, television, and new media like real people and places (1. paperback ed. ed.). CSLI Publ, Stanford, Calif.

[28] Maike A. J. Roubroeks, Jaap R.C. Ham, and Cees J.H. Midden. 2012. Investigating the media equation hypothesis: do we really see computer agents as human-like? https://fisher.osu.edu/blogs/persuasive2011/ 6th International Conference on Persuasive Technology (PERSUASIVE 2011), PERSUASIVE 2011 ; Conference date: 02-06-2011 Through 05-06-2011.

[29] Astrid Schepman and Paul Rodway. 2020. Initial validation of the general attitudes towards Artificial Intelligence Scale. Computers in Human Behavior Reports 1 (2020), 100014. https://doi.org/10.1016/j.chbr.2020.100014

[30] Jakub Złotowski, Diane Proudfoot, Kumar Yogeeswaran, and Christoph Bartneck. 2015. Anthropomorphism: Opportunities and Challenges in Human-Robot Interaction. International fournal of Social Robotics 7, 3 (2015), 347-360. https: //doi.org/10.1007/s12369-014-0267-6 